\title{
Non-Alcoholic Fatty Liver Disease and Hepatocellular Cancer: A Systematic Review
}

\author{
Donna L. White, $\mathrm{PhD}^{1,2,3}$, Fasiha Kanwal, MD, MSHS ${ }^{1,2,3}$, and Hashem B. El-Serag, MD, \\ MPH $^{1,2,3}$ \\ ${ }^{1}$ Clinical Epidemiology and Outcomes Program, Houston VA Health Services Research and \\ Development Center of Excellence, Michael E. DeBakey Veterans Affairs Medical Center, \\ Houston, Texas \\ ${ }^{2}$ Section of Gastroenterology and Hepatology, Department of Medicine, Baylor College of \\ Medicine and Michael E. DeBakey Veterans Affairs Medical Center, Houston, Texas
}

${ }^{3}$ Section of Health Services Research, Department of Medicine, Baylor College of Medicine and Michael E. DeBakey Veterans Affairs Medical Center, Houston, Texas

\section{Abstract \\ Background-Non-alcoholic fatty liver disease (NAFLD) has been implicated as a possible cause of hepatocellular carcinoma (HCC) in several general review articles. We performed the first systematic review of the epidemiologic literature.}

\begin{abstract}
Methods-We searched PubMed for original reports published between 1/1992-12/2011 evaluating the association between NAFLD, non-alcoholic steatohepatitis (NASH) and cryptogenic cirrhosis (CC) presumptively NASH-related and the risk of HCC. Studies were categorized as offering potential direct evidence (e.g., cohort studies) or indirect evidence (e.g., case-control or cross-sectional studies or case-series) of an association.

Results-A total of 17 cohort studies [ 3 population-based, 9 clinic-based (6 limited to cirrhotics), and 5 natural history], 18 case-control and cross-sectional studies, and 26 case-series were study-eligible. NAFLD or NASH cohorts with few or no cirrhosis cases demonstrated a minimal HCC risk (cumulative HCC mortality between $0 \%-3 \%$ for study periods up to two decades). Consistently increased risk was observed in NASH-cirrhosis cohorts (cumulative incidence between $2.4 \%$ over 7 -years to $12.8 \%$ over 3 -years). However, HCC risk was substantially lower in NASH-cirrhosis (NASH-C) cohorts than in HCV-related cirrhosis cohorts. The determinants of elevated risk among NASH-C cohorts were unclear as most studies were underpowered to perform multivariate analysis.
\end{abstract}

Conclusions-This systematic review shows that despite several limitations, the epidemiologic evidence supports an association between NAFLD or NASH and an increased HCC risk that seems to be predominantly limited to individuals with cirrhosis.

(C) 2012 The American Gastroenterological Association. Published by Elsevier Inc. All rights reserved

Correspondence author: Donna L. White, PhD Michael E. DeBakey VA Medical Center 2002 Holcombe Blvd. (MS 152), Houston, Texas77030dwhite1@bcm.edu.

Publisher's Disclaimer: This is a PDF file of an unedited manuscript that has been accepted for publication. As a service to our customers we are providing this early version of the manuscript. The manuscript will undergo copyediting, typesetting, and review of the resulting proof before it is published in its final citable form. Please note that during the production process errors may be discovered which could affect the content, and all legal disclaimers that apply to the journal pertain.

Conflict of Interest Statement: The authors declare no conflict of interest. The U.S. Department of Veteran Affairs, the National Institutes of Health, and the National Institute of Diabetes and Digestive and Kidney Disease played no role in design, implementation, analysis, interpretation or decision to report these results. 


\section{Keywords}

epidemiology; hepatology; gastroenterology; endocrinology; obesity; metabolic syndrome

\section{Introduction}

Rising hepatocellular carcinoma (HCC) incidence rates have been observed in many Western countries, with HCC a rapidly increasing cause of cancer-related deaths in the United States ${ }^{1}$. Hepatitis $\mathrm{C}$ is the most common etiologic risk factor for $\mathrm{HCC}$ within western countries, accounting for 30-50\% of cases, followed by alcohol-related liver disease (10$20 \%$ ) and hepatitis B (10-15\%). Genetic disorders (e.g., Wilson's disease, alpha-1 antitrypsin deficiency, and hereditary hemochromatosis) account for only a very small proportion of cases. HCC arising in the background of cryptogenic liver disease accounts for another $15-50 \%$ of HCC cases in the U.S. ${ }^{2}$

Non-alcoholic fatty liver disease (NAFLD) has been proposed as the underlying cause of most cases of cryptogenic cirrhosis (CC). NAFLD and its more advanced clinical manifestation, non-alcoholic steatohepatitis (NASH), typically occur in patients with key features of the metabolic syndrome including visceral adiposity and insulin resistance. Coincident with large secular increases in both obesity and diabetes, NAFLD is now the leading cause of chronic liver disease in the U.S. ${ }^{3}$

Over the last two decades there has been considerable growth in the literature evaluating the association between NAFLD, NASH or CC and HCC. However, the only systematic reviews and meta-analyses in this area evaluated the association between NAFLD/NASH and overall liver disease-related mortality without distinguishing between cirrhosis and HCC. ${ }^{4,5}$ The association between NAFLD and HCC remains unclear; studies have arrived at different findings as to the presence of an association and the magnitude or determinants of such as an association.

We performed a systematic review of the literature on the association between NAFLD/ NASH and HCC reported in: (1) longitudinal studies on HCC in adults with NAFLD/NASH or cryptogenic cirrhosis (CC) presumptively NAFLD/NASH-related; (2) cross-sectional and case-control studies that examined the association between diabetes or obesity and HCC ascribed to NAFLD/NASH or CC; and (3) case-reports and case-series which described a HCC case-group with etiology ascribed NASH/NAFLD or CC. Our goals were to critically review and synthesize the collective literature and also to identify potential gaps that may be addressed by future research studies.

\section{Methods}

We followed published guidelines for the conduct and reporting of systematic reviews. ${ }^{6} \mathrm{We}$ performed a structured keyword search in PubMed to identify original research reports published in print or online in peer-reviewed journals reporting results in English between 1/1/1992 and 12/31/2011 that evaluated the association between NAFLD/NASH or CC and HCC either directly (e.g., population- and clinic-based cohort studies) or indirectly (e.g., cross-sectional and case-control studies and case-series/reports). Eligible studies had to have data to calculate an estimate of HCC incidence or mortality for cohort studies, relative risk of HCC or prevalence of diabetes or obesity in HCC cases attributed to NAFLD/NASH or $\mathrm{CC}$ and in a comparison group with another type of liver disease for case-control and crosssectional studies, or prevalence of obesity or diabetes for case-reports/case-series. 
Our search included all combinations of individual terms presented in Appendix 1. We also performed ancestry searches by reviewing the bibliography of all retrieved manuscripts as well as of relevant meta-analyses and systematic and narrative reviews on NAFLD/NASH to identify additional studies not identified by our keyword searches.

We excluded publications that: did not report original research findings (e.g., editorials) or were published as abstracts or letters; were performed in pediatric cohorts; had data on participants only post-hepatic transplant, post-bariatric surgery, or after other clinical interventions (e.g., pharmaceutical trials); did not differentiate HCC incidence or mortality from either overall or liver disease-related incidence or mortality; included cryptogenic liver disease cases where viral hepatitis and alcohol-related disease were not specifically excluded; did not report or have data to calculate requisite measures of effect (e.g., cumulative HCC incidence rate in cohort studies or prevalence of diabetes and obesity in case-control studies or case-reports), provided outcome data for only a non-random subset of the baseline cohort, or included data on only secondary or recurrent HCC. We also excluded studies published prior to 1992 because of lack of serological test to specifically exclude the hepatitis $\mathrm{C}$ virus as an alternate cause for observed liver disease.

We applied additional study design eligibility criteria to help assure the internal validity of our review. These additional criteria were: 1 ) case-control and cross-sectional studies had to have a minimum of $5 \mathrm{HCC}$ cases attributed to NAFLD/NASH with at least one control group with HCC attributed to another cause of liver disease to plausibly allow comparisons of at least baseline prevalence or risk between groups as well as data on prevalence of diabetes or obesity in both the case and control groups; 2) cohort studies had to have a NAFLD/NASH or cryptogenic liver disease cohort followed collectively for HCC incidence or mortality and meet sample size requirements (a minimum cohort sample size of 20 and study follow-up period of 3 years if restricted to cirrhosis at baseline or a minimum cohort sample size of 75 with 10 years follow-up if not restricted to cirrhosis at baseline as minimum plausible for HCC to develop); and 3) case-reports or case-series had to have minimum reporting of at least one cardinal feature of the metabolic syndrome in $\mathrm{HCC}$ cases attributed to NAFLD/NASH or cryptogenic disease. If a case-control or cohort study did not meet minimum eligibility criteria for those designs, if adequate data was provided on individual HCC cases, they were included as case-series or case-reports instead.

When more than one eligible report was available for the same study population, we included either the most recent or the largest study unless an earlier study contained more detailed data on comorbidities and outcomes. However, if two separate study designs were employed to evaluate the same underlying target population (e.g., case-control and population-based cohort designs), or if two studies utilized the same NAFLD/NASH or cryptogenic cases but had different comparison groups (e.g., HCV-related and alcoholrelated), results from each study are reported.

Two independent reviewers (DW and FK) abstracted data from eligible reports using a structured data collection template that interrogated data on patient sociodemographic and clinical characteristics as well as on study design (e.g., source of cases and any comparison groups, study country and performance dates, and a general synopsis of criteria used to define NAFLD/NASH or cryptogenic disease). All results are provided in summary tables stratified according to study design (cohort studies, case-control and cross-sectional studies, and case-report and case-series). 


\section{Results}

Our combined PubMed keyword and review article ancestry searches identified $>14,000$ citations (see study flow diagram Figure 1). Among the 265 full citations reviewed, the most common reason for exclusion was report did not contain specific results for HCC or did not adequately distinguish HCC attributed to NAFLD/NASH or CC from HCC attributed to other causes (e.g., alcohol-related HCC) or other health outcomes (e.g., overall liver-related mortality or total mortality). A total of 17 cohort studies, 18 case-control and cross-sectional studies, and 26 case-reports/series were eligible for inclusion in our review.

\section{Cohort studies}

Among the 17 eligible cohort studies, 3 were population-based studies with a case and a comparison cohort, ${ }^{7-9} 9$ were clinic-based cohort studies with a comparison cohort (6 of which were limited to patients with cirrhosis), ${ }^{10-18}$ and 5 were natural history cohorts without a comparison cohort. ${ }^{19-23}$

Population-based cohort studies with a comparison group-Two populationbased cohort studies were from the U.S. and dealt with all comers with presumed NAFLD ${ }^{8,9}$, while the third study from Denmark evaluated only those with cryptogenic cirrhosis (CC). ${ }^{7}$ (Table 1A) One U.S. population-based study identified NAFLD using data collected in the Nutrition Health and Exercise Study (NHANES). ${ }^{9}$ NAFLD was defined as presence of elevated liver enzymes measured at baseline in the absence of other causes of liver disease. In this study, none of the 817 NAFLD cases or the 10,468 study eligible normal controls without liver disease died from HCC during the median 8.7 year follow-up period. (Table 2A) The validity of their NAFLD/NASH definition was not examined.

The second U.S. population-based study used a national health insurance database to identify NAFLD/NASH (via an ICD-code based algorithm) in >729,000 enrolled studyeligible participants. ${ }^{8}$ (Table 1A) The cumulative HCC incidence in the 6 years between 2002 and 2008 was $0.3 \%$ in NAFLD/NASH cases compared to $0.6 \%$ in those with HCVrelated liver disease. (Table 2A) The validity of the NAFLD/NASH definition was again not examined.

The third study performed in Denmark utilized nationwide hospitalization data and reported that $1.9 \%$ of the 2,430 cirrhosis cases who had a hospital discharge for CC developed HCC during the follow-up period, which ranged between 5.5 years for males and 5.9 years for females. ${ }^{7}$ (Table 2A) The standardized incidence rate (SIR) for HCC was lower in those with CC than those with alcohol-related cirrhosis ( $\mathrm{SIR}=43 / 100,000$ and $71 / 100,000$ personyears for CC-related HCC and alcoholic cirrhosis-related HCC, respectively), but was the same as that observed for HCV-related HCC.

None of these population-based cohort studies reported the proportion of participants with biopsy-confirmed features of NAFLD/NASH. (Table 1A) Sociodemographic and clinical characteristics of the resulting HCC cases attributed to NAFLD/NASH were also generally not reported. Finally, neither of the two studies where HCC cases occurred during the follow-up period included multivariate analyses to identify risk factors for HCC.

\section{Clinic-based cohort studies of cirrhosis potentially NASH-related with} comparison cohort-All six studies in this group evaluated HCC risk in a cohort with cirrhosis or advanced fibrosis with clinically-confirmed NASH or cryptogenic cirrhosis (presumptively NASH-related) and in a comparison cohort with HCV-related cirrhosis. ${ }^{10-15}$ (Table 1A) Diabetes was more prevalent in all NASH cirrhosis and CC cohorts compared with their comparison HCV-related cirrhosis cohorts except in one study. (Table 2A) 
Obesity and dyslipidemia were uniformly higher in NASH cirrhosis and CC cohorts compared to HCV-related cohorts.

Five studies reported cumulative HCC incidence for the NASH-cirrhosis or CC cohorts and the comparison HCV cirrhosis cohorts. ${ }^{10,12-15}$ (Table 2A) The cumulative HCC incidence in NASH-cirrhosis or CC cohorts varied widely (range: $2.4 \%-38 \%$ ) over generally modest follow-up periods (range: $3.2-10$ years). However, cumulative HCC incidence was always lower in NASH cirrhosis or CC than in the HCV-related cirrhosis (i.e., internal control) cohorts except for one sub-group comparison in a single study from France (29.6\% vs. $19.5 \%$ cumulative HCC incidence in overweight CC and HCV-related cirrhosis subgroups over a 3.4 year follow-up period, respectively). ${ }^{10}$ The single study reporting only cumulative HCC mortality also found a lower mortality rate with CC than in the HCV cohort $(6.7 \%$ vs. $17.0 \%$ cumulative 25 -year HCC mortality among the CC and HCV-related cirrhosis cohorts, respectively). ${ }^{11}$

Information on the sociodemographic and clinical characteristics for the incident HCC cases attributed to NASH-cirrhosis or CC were generally not reported. Three of four studies which performed multivariate analyses found no independent risk factors for $\mathrm{HCC}$ in the background of NASH-cirrhosis or CC. ${ }^{11,13,15}$ (data not shown) However, a recent study performed in the U.S. found that NASH-cirrhosis cases with any type of alcohol consumption had a significant 3.6-fold excess HCC risk compared to NASH-cirrhosis cases who were never drinkers. ${ }^{14}$ (data not shown)

In summary, although the cumulative HCC incidence and mortality rates were variably elevated over observed study periods, the relative HCC risk was almost universally lower in $\mathrm{NASH}$-cirrhosis and $\mathrm{CC}$ cohorts relative to their respective cirrhosis comparison cohorts (range overall excess HCC risk with HCV vs. NASH-C or CC: $58 \%-172 \%$ ).

Clinic-based cohort study of NAFLD-NASH (not cirrhosis restricted) and a comparison cohort-Three clinic-based cohort studies evaluated HCC risk in a cohort with NAFLD or NASH, two with biopsy-confirmed NAFLD/NASH cases ( $\mathrm{n}=1$ Denmark, $\mathrm{n}=1$ Sweden) ${ }^{16,17}$ and one with ultrasound-confirmed NAFLD/NASH cases (Japan). ${ }^{18}$ (Table 1A)

The cumulative HCC incidence reported in these studies varied widely. In the Danish cohort study, none of the 170 subjects in the NAFLD cohort without significant fibrosis at baseline developed HCC during an average of almost 21 years follow-up compared to $1 \%$ in a cohort with alcohol-related fatty liver. ${ }^{17}$ The Swedish cohort followed for 21 years found 3\%, 6\%, $7 \%$ and $8 \%$ cumulative HCC mortality for the NAFLD, NASH, HCV, and alcohol abuse cohorts, respectively. ${ }^{16}$ The Japanese cohort study was performed in adults aged 60 years or older at baseline and reported 6\% cumulative HCC incidence in the NAFLD/NASH cohort vs. $63 \%$ in the $\mathrm{HCV}$ cohort during the average 8.2 year follow-up period. ${ }^{18}$ (Table $2 \mathrm{~A}$ ) That study also included multivariate analyses that identified older age, smoking and glucose level as significant independent predictors of malignancies in the NAFLD cohort including HCC. (data not shown)

Diabetes was modestly more prevalent in NAFLD/NASH cohorts compared to their respective comparison cohorts with other causes of liver disease (e.g., 34\% in NAFLD vs. $28 \% \mathrm{HCV}$ in the Japanese cohort study). (Table 2) NAFLD cohort members were also more likely to be obese (e.g., $69 \%$ vs. $20 \%$ obesity prevalence in the NAFLD vs. HCV comparison cohort in the Danish study). 
In summary, the findings of these three studies show between no HCC occurrence in a NAFLD cohort without significant fibrosis (Danish study) to a small elevated absolute risk of HCC in the NAFLD/NASH cohorts in the other two studies (3-6\%) over study periods ranging between approximately 1-2 decades, with all three demonstrating HCC was less likely to develop in NAFLD/NASH cohorts compared to their respective alcohol- or HCVrelated comparison cohorts.

Clinic based cohorts without control groups (Natural history cohort) studiesFive natural history cohort studies were included ( $\mathrm{n}=2$ Japan, $\mathrm{n}=1$ Sweden, and $\mathrm{n}=2$ U.S.). ${ }^{19-23}$ (Table 1B) Three reported cumulative HCC mortality rates, which ranged between $0.25 \%$ in the Rochester, Minnesota cohort of NAFLD/NASH cases or CC cases with features of metabolic syndrome followed for an average of 7.6 years, ${ }^{20} 1 \%$ in the Cleveland Clinic cohort which was followed an average of 8.3 years and included $26 \%$ with ballooning degeneration and fibrosis at baseline, ${ }^{19}$ and $2.3 \%$ in the Swedish cohort study which included cases with fatty infiltration and persistent abnormal liver tests at baseline (only $3.4 \%$ cirrhotic) and followed on average for 13 years. ${ }^{21}$ (Table 2B) The Rochester, Minnesota study, while population-based, did not provide a population standardized mortality risk estimate given only $2 \mathrm{HCC}$ cases were recorded (one in a NAFLD case and one in a CC case). ${ }^{20}$

The rates reported in the Japanese studies ranged between a 7.6\% 5-year cumulative incidence rate in a cohort of 118 cases with advanced NASH-related fibrosis or cirrhosis at baseline ( $41 \%$ male and $43 \%$ diabetic) ${ }^{22}$ vs. a $0.25 \%$ cumulative incidence rate in a cohort of $>6,500$ ultrasound confirmed NAFLD/NASH cases ( $88 \%$ male and $8 \%$ diabetic) and followed on average of 5.6 years. ${ }^{23}$ Only the larger study identified significant predictors NAFLD-related HCC in multivariate analysis (BMI, AST, platelet count and diabetes). (data not shown)

\section{Case-control and cross-sectional studies}

The largest number of eligible studies in this review were case-control and cross-sectional studies ( $\mathrm{n}=18),{ }^{8,22,24-39}$ with 8 performed in Asia ( $\mathrm{n}=7$ in Japan), 6 in Europe and 4 in the U.S. (Table 1C) All compared a case group with HCC attributed to NASH/NAFLD or to CC presumptively NAFLD-related with at least one control group, typically with HCC attributed to another cause of liver disease $(n=16)$, though two had only NASH-related cirrhosis controls without HCC as their sole comparison or control group. Most ( $\mathrm{n}=15)$ also utilized case and control groups identified from single medical centers, with 9 studies having biopsy-based confirmation of fatty liver disease in $>80 \%$ of their NAFLD/NASH-related HCC cases (7 with verification in 100\% of cases) either prior to or associated with HCC diagnosis or treatment.

Among studies reporting results for each gender, NAFLD/NASH or cryptogenic HCC cases were generally more likely to be male than $\mathrm{HCC}$ controls with other underlying causes of liver disease ( 8 of 10 studies). (Table 2C) Most studies included NAFLD/NASH or cryptogenic HCC cases either entirely or predominantly comprised of one ethnic/racial group (e.g., 83-100\% White or 100\% Japanese).

Prevalence of cirrhosis among HCC cases attributed to NAFLD/NASH in studies not restricted to cirrhotics ranged between $36 \%$ and $90 \%$, with 9 of 12 studies reporting on cirrhosis prevalence reporting rates $\geq 70 \%$. (Table 1C) Most studies reported significantly higher diabetes prevalence in the NAFLD/NASH- or CC-related disease related HCC cases than in HCC control groups with other types of liver disease. (Table 2C) Diabetes was also more prevalent in HCC-NASH or CC-related HCC cases than in NASH controls without HCC in two of three studies with that control group ( $86 \%$ vs. $52 \%$, p=0.02; $74 \%$ vs. $43 \%$, 
$\mathrm{p}=0.002$; and $71 \%$ vs. $73 \%$, NS, respectively). Many studies also reported higher obesity prevalence or greater BMI in HCC-NASH or HCC-CC cases than that observed in HCC control groups with other types of liver disease, though fewer comparisons reached statistical significance.

\section{Case-reports and Case-series}

We identified 26 case-reports and case-series comprising a total of $257 \mathrm{HCC}$ cases attributed to NAFLD/NASH or metabolic syndrome (presumptive fatty liver disease). ${ }^{40-65}$ (Appendix 2) The majority of the reports were from Japan $(n=15)$, while 5 were from Europe, 3 from the U.S., and 3 from Brazil. Most included data on prevalence of two primary features of the metabolic syndrome, diabetes and obesity, with overall estimated sample-size weighted prevalence of $58 \%$ and $63 \%$ for diabetes and obesity, respectively. Approximately $60 \%$ of cases had cirrhosis either prior to or at HCC diagnosis. Only five reports specifically noted that their HCC cases had presence of biopsy-confirmed features of NAFLD/NASH prior to HCC diagnosis. (Appendix 2)

None of the studies included in this review explicitly assessed for potential selection or information biases that may have influenced the validity and reliability of their reported findings, including for potential case misspecification bias that might occur in studies which solely relied on diagnostic code searches to identify NAFLD/NASH cases.

\section{Discussion}

This systematic review shows that despite several limitations, the epidemiologic evidence supports an association between NAFLD/NASH and an increased HCC risk that seems to be predominantly limited to those with cirrhosis. The studies that followed NAFLD or NASH cohorts with either few or no cirrhosis cases at baseline were consistent in showing that NAFLD/NASH was associated with a minimal HCC risk; cumulative HCC mortality rates ranged between $0 \%-3 \%$ in NAFLD/NASH cohorts, with 5 of 7 studies reporting $0 \%-1 \%$ cumulative $\mathrm{HCC}$ mortality over study periods ranging from 5.6-21 years. In contrast, cirrhosis related to NASH consistently signaled increased HCC risk, with cumulative HCC incidence ranging between $2.4 \%$ in a study that combined clinic-based cohorts from 4 countries with median follow-up of 7.2 years ${ }^{15}$ and $12.8 \%$ in a single clinic-based cohort from the U.S. with 3.2 year median follow-up. ${ }^{14} \mathrm{HCC}$ risk was universally lower in the $\mathrm{NASH}$-cirrhotic cohorts than in $\mathrm{HCV}$-related cirrhosis comparison cohorts followed over similar timeframes. However, the determinants of this elevated HCC risk among NASHcirrhosis cases were not clear as most studies were underpowered to perform multivariate analysis. Indirect evidence of a NAFLD-HCC association was provided by numerous casecontrol and cross-sectional studies showing universally higher prevalence of both diabetes and obesity among patients with HCC attributed to NASH or cryptogenic disease presumptively fatty liver disease-related compared to controls with $\mathrm{HCC}$ due to other causes of liver disease (e.g., 2.3-8.3 fold excess diabetes risk and 3.6-5.7 fold excess obesity risk in $\mathrm{NAFLD/NASH/CC-related} \mathrm{HCC} \mathrm{cases} \mathrm{vs.} \mathrm{their} \mathrm{respective} \mathrm{HCV-related} \mathrm{HCC} \mathrm{comparison}$ groups).

Direct comparison of findings from analytic studies that belong to different design categories (e.g., population-based cohort, clinic-based natural history studies, and casecontrol or cross-sectional studies) is generally not possible. Furthermore, differences in the risk estimates among studies that belonged to the same design category may be attributed to their source population or their inclusion of cases with more advanced disease at baseline. Therefore, it is important that individual study findings be discussed and qualified according to study design. 
Cohort studies, particularly those that are population-based, prospective, have a priori welldefined exposure and outcome groups, and have sufficient sample size and longitudinal follow-up periods, are considered the strongest direct observational epidemiologic design in support of a potential causal association. However, none of the cohort studies included in this review met all of these design criteria. Low numbers of HCC cases in many of these cohort studies often resulted in imprecise risk estimates and an inability to perform meaningful multivariate analyses. Several of the largest cohort studies relied on diagnostic codes (e.g., ICD-9 571.8, 571.9, 573.4, 573.8, 573.9). However, they may have potentially substantial misclassification rates because none of these codes are specific for NAFLD/ $\mathrm{NASH}$, with none of the studies reporting on efforts to validate codes against clinical data. Additionally, all the studies except for NHANES evaluated NASH/NAFLD/CC that was ascertained in the setting of clinical practice. The generalizability of these clinic-based findings to population or community-based NAFLD/NASH screening cohorts is unclear.

Cross-sectional and case-control studies have evaluated the association between NAFLD/ NASH and HCC indirectly by concomitantly examining diabetes and obesity, primary features of the metabolic syndrome and also well-established NAFLD risk factors. Diabetes prevalence was uniformly greater in the NASH/NAFLD case-groups compared to their respective control groups with other types of chronic liver disease. Similarly, obesity prevalence was significantly greater in all NASH/NAFLD case groups. However, in addition to their inherent inability to establish temporality necessary to firmly establish causality, cross-sectional and case-control studies are also limited by the difficulty in ascertaining the exposure (histopathological features for confirmed NAFLD/NASH diagnosis) once cirrhosis is established. Moreover, these studies are limited by the possibility of reverse causality in the case of diabetes. Most of the studies that reported on obesity used BMI but provided no information on potentially more relevant parameters of obesity such as waist circumference, $\%$ body fat or visceral adiposity. Measurement and reporting on additional aspects of both insulin resistance and adiposity, including disease severity, duration and treatment may be useful in identifying sub-groups at particularly increased risk of progression to HCC in the background of NAFLD/NASH. Although qualified given the limited numbers of women with $\mathrm{HCC}$ in most studies, there was also a potentially suggestive finding regarding gender, with NAFLD/NASH-and cryptogenic-related HCC case groups often having an even greater preponderance of males than similar HCC case groups attributed to other causes of liver disease. Given women constitute a substantial proportion of NAFLD/NASH cases seen in the U.S., ${ }^{66}$ especially at older ages, this potentially particularly enhanced excess HCC risk among males with NAFLD/NASH bears further examination.

Additional indirect evidence suggestive of an association comes from multiple case-reports and case-series describing well-documented NAFLD/NASH patients who developed HCC. Although the prevalence of cirrhosis among HCC cases in case-control and cross-sectional studies was $>70 \%$, these studies nonetheless also suggest that a substantial minority of NAFLD/NASH-related HCC cases develop in the absence of clinically manifest cirrhosis. However, the interpretation of findings from these reports must be qualified because of the small number of cases.

This systematic review has several limitations. The search for PubMed indexed papers published in peer-reviewed journals in English may have missed some relevant papers in this area. However, we believe our comprehensive search strategy likely captured most published original research, while assuring a minimum standard of comparability and quality of reported data among studies thus enhancing our review's internal validity. We did not use quality scores to rate individual studies included this review as they can introduce bias of unknown dimensions when employed in systematic reviews and meta-analyses of observational studies. ${ }^{67}$ Instead, we categorized studies based on meaningful differences in 
design and explained the overall strengths and limitations of each design. We included studies that had HCC cases attributed to cryptogenic cirrhosis where other major causes of liver disease had been explicitly excluded as this is widely considered to most usually be associated with fatty liver disease. Many studies were based on results from routine clinical care and thus most did not have confirmatory biopsy data on their entire study sample. Therefore, it is possible that some NAFLD/NASH cohorts may have contained some nearly cirrhotic or well-compensated cirrhosis cases. We were not able to reliably or validly calculate annual or age- or gender-adjusted estimates of HCC incidence in multiple studies because adequate data was rarely provided (e.g., person-years at risk by gender or age-group and losses to follow-up). We were also unable to perform meta-analyses to obtain pooled estimators for incidence or prevalence rate of NAFLD/NASH-associated HCC as there were too few comparably conducted studies that reported similar effect measures. Finally, we did not evaluate survival differences between NAFLD-related HCC and other types of HCC, although some studies suggested that NAFLD-related HCC may be diagnosed later or at a more advanced stage. ${ }^{24,26,35,37}$

The currently available data do not support routine HCC surveillance among general cohorts of NAFLD or NASH patients who do not have cirrhosis. For the latter group, while the risk of HCC may be increased, this is only one aspect of an otherwise complicated progression that bears further examination from an epidemiologic as well as decision analytic perspective.

Our review has also identified several key gaps in current knowledge, including the lack of data on associations in ethnic minority populations including in Hispanics in whom NAFLD is disproportionately common, ${ }^{68}$ and in African Americans in whom HCC risk incidence rates are higher and increasing more rapidly than in whites. ${ }^{69}$ Additional research performed in substantially larger cohorts with longer follow-up is needed to identify risk factors that may be associated with progression to HCC in particular sub-groups of interest, including in the absence of clinically manifest cirrhosis.

\section{Acknowledgments}

This material is based upon work supported in part by a VA Clinical Research and Development Merit Review Award (H-22934, PI: H. El-Serag, MD, MPH), the National Institute of Diabetes Digestive and Kidney Diseases (K01 DK078154-03 and K24 DK081736-01, PIs, D. White and H. El-Serag, respectively), the Texas Medical Center Digestive Disease Center (P30 Center Grant DK56338), and the Houston VA HSR\&D Center of Excellence (HFP90-020). We thank Adrian Rideau, BA for her administrative assistance.

\section{Abbreviations}

$\begin{array}{ll}\text { HCC } & \text { hepatocellular carcinoma } \\ \text { NAFLD } & \text { non-alcoholic fatty liver disease } \\ \text { NASH } & \text { non-alcoholic steatosis } \\ \text { CC } & \text { cryptogenic cirrhosis } \\ \text { LD } & \text { liver disease }\end{array}$

\section{Reference List}

(1). El-Serag HB, Davila JA, Petersen NJ, McGlynn KA. The continuing increase in the incidence of hepatocellular carcinoma in the United States: an update. Ann Intern Med. 2003; 139:817-823. [PubMed: 14623619] 
(2). El-Serag HB. Epidemiology of hepatocellular carcinoma in USA. Hepatol Res. 2007; 37(Suppl 2):S88-S94. [PubMed: 17877502]

(3). Adams LA, Lindor KD. Nonalcoholic fatty liver disease. Ann Epidemiol. 2007; 17:863-869. [PubMed: 17728149]

(4). Musso G, Gambino R, Cassader M, Pagano G. Meta-analysis: natural history of non-alcoholic fatty liver disease (NAFLD) and diagnostic accuracy of non-invasive tests for liver disease severity. Ann Med. 2011; 43:617-649. [PubMed: 21039302]

(5). Lazo M, Hernaez R, Bonekamp S, et al. Non-alcoholic fatty liver disease and mortality among US adults: prospective cohort study. BMJ. 2011; 343:d6891. [PubMed: 22102439]

(6). Moher D, Liberati A, Tetzlaff J, Altman DG, PRISMA Group. Preferred reporting items for systematic reviews and meta-analyses: the PRISMA statement. Ann Intern Med. 2009; 151:2649. W64. [PubMed: 19622511]

(7). Sorensen HT, Friis S, Olsen JH, et al. Risk of liver and other types of cancer in patients with cirrhosis: a nationwide cohort study in Denmark. Hepatology. 1998; 28:921-925. [PubMed: 9755226]

(8). Sanyal A, Poklepovic A, Moyneur E, Barghout V. Population-based risk factors and resource utilization for HCC: US perspective. Curr Med Res Opin. 2010; 26:2183-2191. [PubMed: 20666689]

(9). Ong JP, Pitts A, Younossi ZM. Increased overall mortality and liver-related mortality in nonalcoholic fatty liver disease. J Hepatol. 2008; 49:608-612. [PubMed: 18682312]

(10). Ratziu V, Bonyhay L, Di M, V, et al. Survival, liver failure, and hepatocellular carcinoma in obesity-related cryptogenic cirrhosis. Hepatology. 2002; 35:1485-1493. [PubMed: 12029634]

(11). Sanyal AJ, Banas C, Sargeant C, et al. Similarities and differences in outcomes of cirrhosis due to nonalcoholic steatohepatitis and hepatitis C. Hepatology. 2006; 43:682-689. [PubMed: 16502396]

(12). Kojima H, Sakurai S, Matsumura M, et al. Cryptogenic cirrhosis in the region where obesity is not prevalent. World J Gastroenterol. 2006; 12:2080-2085. [PubMed: 16610061]

(13). Yatsuji S, Hashimoto E, Tobari M, Taniai M, Tokushige K, Shiratori K. Clinical features and outcomes of cirrhosis due to non-alcoholic steatohepatitis compared with cirrhosis caused by chronic hepatitis C. J Gastroenterol Hepatol. 2009; 24:248-254. [PubMed: 19032450]

(14). Ascha MS, Hanouneh IA, Lopez R, Tamimi TA, Feldstein AF, Zein NN. The incidence and risk factors of hepatocellular carcinoma in patients with nonalcoholic steatohepatitis. Hepatology. 2010; 51:1972-1978. [PubMed: 20209604]

(15). Bhala N, Angulo P, van der PD, et al. The natural history of nonalcoholic fatty liver disease with advanced fibrosis or cirrhosis: an international collaborative study. Hepatology. 2011; 54:12081216. [PubMed: 21688282]

(16). Soderberg C, Stal P, Askling J, et al. Decreased survival of subjects with elevated liver function tests during a 28-year follow-up. Hepatology. 2010; 51:595-602. [PubMed: 20014114]

(17). Dam-Larsen S, Becker U, Franzmann MB, Larsen K, Christoffersen P, Bendtsen F. Final results of a long-term, clinical follow-up in fatty liver patients. Scand J Gastroenterol. 2009; 44:12361243. [PubMed: 19670076]

(18). Arase Y, Kobayashi M, Suzuki F, et al. Difference in malignancies of chronic liver disease due to non-alcoholic fatty liver disease or hepatitis C in Japanese elderly patients. Hepatol Res. 2012; 42:264-272. [PubMed: 22175908]

(19). Matteoni CA, Younossi ZM, Gramlich T, Boparai N, Liu YC, McCullough AJ. Nonalcoholic fatty liver disease: a spectrum of clinical and pathological severity. Gastroenterology. 1999; 116:1413-1419. [PubMed: 10348825]

(20). Adams LA, Lymp JF, St SJ, et al. The natural history of nonalcoholic fatty liver disease: a population-based cohort study. Gastroenterology. 2005; 129:113-121. [PubMed: 16012941]

(21). Ekstedt M, Franzen LE, Mathiesen UL, et al. Long-term follow-up of patients with NAFLD and elevated liver enzymes. Hepatology. 2006; 44:865-873. [PubMed: 17006923]

(22). Hashimoto E, Yatsuji S, Tobari M, et al. Hepatocellular carcinoma in patients with nonalcoholic steatohepatitis. J Gastroenterol. 2009; 44(Suppl 19):89-95. [PubMed: 19148800] 
(23). Kawamura Y, Arase Y, Ikeda K, et al. Large-scale long-term follow-up study of Japanese patients with non-alcoholic Fatty liver disease for the onset of hepatocellular carcinoma. Am J Gastroenterol. 2012; 107:253-261. [PubMed: 22008893]

(24). Marrero JA, Fontana RJ, Su GL, Conjeevaram HS, Emick DM, Lok AS. NAFLD may be a common underlying liver disease in patients with hepatocellular carcinoma in the United States. Hepatology. 2002; 36:1349-1354. [PubMed: 12447858]

(25). Bugianesi E, Leone N, Vanni E, et al. Expanding the natural history of nonalcoholic steatohepatitis: from cryptogenic cirrhosis to hepatocellular carcinoma. Gastroenterology. 2002; 123:134-140. [PubMed: 12105842]

(26). Regimbeau JM, Colombat M, Mognol P, et al. Obesity and diabetes as a risk factor for hepatocellular carcinoma. Liver Transpl. 2004; 10:S69-S73. [PubMed: 14762843]

(27). Abe H, Yoshizawa K, Kitahara T, Aizawa R, Matsuoka M, Aizawa Y. Etiology of non-B non-C hepatocellular carcinoma in the eastern district of Tokyo. J Gastroenterol. 2008; 43:967-974. [PubMed: 19107341]

(28). Paradis V, Zalinski S, Chelbi E, et al. Hepatocellular carcinomas in patients with metabolic syndrome often develop without significant liver fibrosis: a pathological analysis. Hepatology. 2009; 49:851-859. [PubMed: 19115377]

(29). Malik SM, Gupte PA, de Vera ME, Ahmad J. Liver transplantation in patients with nonalcoholic steatohepatitis-related hepatocellular carcinoma. Clin Gastroenterol Hepatol. 2009; 7:800-806. [PubMed: 19281869]

(30). Sorrentino P, D'Angelo S, Ferbo U, Micheli P, Bracigliano A, Vecchione R. Liver iron excess in patients with hepatocellular carcinoma developed on non-alcoholic steato-hepatitis. J Hepatol. 2009; 50:351-357. [PubMed: 19070395]

(31). Ertle J, Dechene A, Sowa JP, et al. Non-alcoholic fatty liver disease progresses to hepatocellular carcinoma in the absence of apparent cirrhosis. Int J Cancer. 2011; 128:2436-2443. [PubMed: 21128245]

(32). Tokushige K, Hashimoto E, Yatsuji S, et al. Prospective study of hepatocellular carcinoma in nonalcoholic steatohepatitis in comparison with hepatocellular carcinoma caused by chronic hepatitis C. J Gastroenterol. 2010; 45:960-967. [PubMed: 20376504]

(33). Cho EJ, Kwack MS, Jang ES, et al. Relative etiological role of prior hepatitis B virus infection and nonalcoholic fatty liver disease in the development of non-B non-C hepatocellular carcinoma in a hepatitis B-endemic area. Digestion. 2011; 84(Suppl 1):17-22. [PubMed: 22156481]

(34). Tokushige K, Hashimoto E, Horie Y, Taniai M, Higuchi S. Hepatocellular carcinoma in Japanese patients with nonalcoholic fatty liver disease, alcoholic liver disease, and chronic liver disease of unknown etiology: report of the nationwide survey. J Gastroenterol. 2011; 46:1230-1237. [PubMed: 21748549]

(35). Takuma Y, Nouso K, Makino Y, et al. Outcomes after curative treatment for cryptogenic cirrhosis-associated hepatocellular carcinoma satisfying the Milan criteria. J Gastroenterol Hepatol. 2011; 26:1417-1424. [PubMed: 21884248]

(36). Yang JD, Harmsen WS, Slettedahl SW, et al. Factors that affect risk for hepatocellular carcinoma and effects of surveillance. Clin Gastroenterol Hepatol. 2011; 9:617-623. [PubMed: 21459158]

(37). Wakai T, Shirai Y, Sakata J, Korita PV, Ajioka Y, Hatakeyama K. Surgical outcomes for hepatocellular carcinoma in nonalcoholic fatty liver disease. J Gastrointest Surg. 2011; 15:1450 1458. [PubMed: 21512848]

(38). Hashimoto E, Taniai M, Kaneda H, et al. Comparison of hepatocellular carcinoma patients with alcoholic liver disease and nonalcoholic steatohepatitis. Alcohol Clin Exp Res. 2004; 28:164S168S. [PubMed: 15318106]

(39). Donadon V, Balbi M, Ghersetti M, et al. Antidiabetic therapy and increased risk of hepatocellular carcinoma in chronic liver disease. World J Gastroenterol. 2009; 15:2506-2511. [PubMed: 19469001]

(40). Cotrim HP, Parise ER, Oliveira CP, et al. Nonalcoholic fatty liver disease in Brazil. Clinical and histological profile. Ann Hepatol. 2011; 10:33-37. [PubMed: 21301007]

(41). Zen Y, Katayanagi K, Tsuneyama K, Harada K, Araki I, Nakanuma Y. Hepatocellular carcinoma arising in non-alcoholic steatohepatitis. Pathol Int. 2001; 51:127-131. [PubMed: 11169153] 
(42). Shimada M, Hashimoto E, Taniai M, et al. Hepatocellular carcinoma in patients with nonalcoholic steatohepatitis. J Hepatol. 2002; 37:154-160. [PubMed: 12076877]

(43). Mori S, Yamasaki T, Sakaida I, et al. Hepatocellular carcinoma with nonalcoholic steatohepatitis. J Gastroenterol. 2004; 39:391-396. [PubMed: 15168253]

(44). Ikeda H, Suzuki M, Takahashi H, et al. Hepatocellular carcinoma with silent and cirrhotic nonalcoholic steatohepatitis, accompanying ectopic liver tissue attached to gallbladder. Pathol Int. 2006; 56:40-45. [PubMed: 16398679]

(45). Cuadrado A, Orive A, Garcia-Suarez C, et al. Non-alcoholic steatohepatitis (NASH) and hepatocellular carcinoma. Obes Surg. 2005; 15:442-446. [PubMed: 15826485]

(46). Hai S, Kubo S, Shuto T, et al. Hepatocellular carcinoma arising from nonalcoholic steatohepatitis: report of two cases. Surg Today. 2006; 36:390-394. [PubMed: 16554999]

(47). Iannaccone R, Piacentini F, Murakami T, et al. Hepatocellular carcinoma in patients with nonalcoholic fatty liver disease: helical CT and MR imaging findings with clinical-pathologic comparison. Radiology. 2007; 243:422-430. [PubMed: 17356175]

(48). Hashizume H, Sato K, Takagi H, et al. Primary liver cancers with nonalcoholic steatohepatitis. Eur J Gastroenterol Hepatol. 2007; 19:827-834. [PubMed: 17873605]

(49). Maeda T, Hashimoto K, Kihara Y, et al. Surgically resected hepatocellular carcinomas in patients with non-alcoholic steatohepatitis. Hepatogastroenterology. 2008; 55:1404-1406. [PubMed: 18795699]

(50). Guzman G, Brunt EM, Petrovic LM, Chejfec G, Layden TJ, Cotler SJ. Does nonalcoholic fatty liver disease predispose patients to hepatocellular carcinoma in the absence of cirrhosis? Arch Pathol Lab Med. 2008; 132:1761-1766. [PubMed: 18976012]

(51). Takamatsu S, Noguchi N, Kudoh A, et al. Influence of risk factors for metabolic syndrome and non-alcoholic fatty liver disease on the progression and prognosis of hepatocellular carcinoma. Hepatogastroenterology. 2008; 55:609-614. [PubMed: 18613418]

(52). Chagas AL, Kikuchi LO, Oliveira CP, et al. Does hepatocellular carcinoma in non-alcoholic steatohepatitis exist in cirrhotic and non-cirrhotic patients? Braz J Med Biol Res. 2009; 42:958962. [PubMed: 19787150]

(53). Kawada N, Imanaka K, Kawaguchi T, et al. Hepatocellular carcinoma arising from non-cirrhotic nonalcoholic steatohepatitis. J Gastroenterol. 2009; 44:1190-1194. [PubMed: 19672551]

(54). Ohki T, Tateishi R, Shiina S, et al. Visceral fat accumulation is an independent risk factor for hepatocellular carcinoma recurrence after curative treatment in patients with suspected NASH. Gut. 2009; 58:839-844. [PubMed: 19174415]

(55). Smith BM, Hussain A, Jacobs M, Merrick HW III. Ruptured hepatocellular carcinoma in a patient with nonalcoholic steatohepatitis. Surg Obes Relat Dis. 2009; 5:510-512. [PubMed: 19342313]

(56). Takuma Y, Nouso K. Nonalcoholic steatohepatitis-associated hepatocellular carcinoma: our case series and literature review. World J Gastroenterol. 2010; 16:1436-1441. [PubMed: 20333782]

(57). Hessheimer AJ, Forner A, Varela M, Bruix J. Metabolic risk factors are a major comorbidity in patients with cirrhosis independent of the presence of hepatocellular carcinoma. Eur J Gastroenterol Hepatol. 2010; 22:1239-1244. [PubMed: 20505515]

(58). Yasui K, Hashimoto E, Komorizono Y, et al. Characteristics of patients with nonalcoholic steatohepatitis who develop hepatocellular carcinoma. Clin Gastroenterol Hepatol. 2011; 9:428433. [PubMed: 21320639]

(59). Stefano JT, de Oliveira CP, Correa-Giannella ML, et al. Decreased immunoexpression of survivin could be a potential marker in human non-alcoholic fatty liver disease progression? Liver Int. 2011; 31:377-385. [PubMed: 21108736]

(60). Orikasa H, Ohyama R, Tsuka N, Eyden BP, Yamazaki K. Lipid-rich clear-cell hepatocellular carcinoma arising in non-alcoholic steatohepatitis in a patient with diabetes mellitus. $\mathrm{J}$ Submicrosc Cytol Pathol. 2001; 33:195-200. [PubMed: 11686402]

(61). Anagnostopoulos GK, Arvanitidis D, Tsiakos S, Margantinis G, Grigoriadis K, Kostopoulos P. Is hepatocellular carcinoma part of the natural history of nonalcoholic steatohepatitis? J Clin Gastroenterol. 2003; 37:88-89. [PubMed: 12811221] 
(62). Gonzalez L, Blanc JF, Sa CA, et al. Obesity as a risk factor for hepatocellular carcinoma in a noncirrhotic patient. Semin Liver Dis. 2004; 24:415-419. [PubMed: 15605309]

(63). Bullock RE, Zaitoun AM, Aithal GP, Ryder SD, Beckingham IJ, Lobo DN. Association of nonalcoholic steatohepatitis without significant fibrosis with hepatocellular carcinoma. J Hepatol. 2004; 41:685-686. [PubMed: 15464253]

(64). Kawai H, Nomoto M, Suda T, et al. Multicentric occurrence of hepatocellular carcinoma with nonalcoholic steatohepatitis. World J Hepatol. 2011; 3:15-23. [PubMed: 21307983]

(65). Ichikawa T, Yanagi K, Motoyoshi Y, et al. Two cases of non-alcoholic steatohepatitis with development of hepatocellular carcinoma without cirrhosis. J Gastroenterol Hepatol. 2006; 21:1865-1866. [PubMed: 17074033]

(66). Bambha K, Belt P, Abraham M, et al. Ethnicity and nonalcoholic fatty liver disease. Hepatology. 2012; 55:769-780. [PubMed: 21987488]

(67). Petitti, DB. Meta-analysis, decision analysis, and cost-effectiveness analysis. Second ed.. Oxford University Press; New York: 2000. Advanced issues in meta-analysis.

(68). Williams CD, Stengel J, Asike MI, et al. Prevalence of nonalcoholic fatty liver disease and nonalcoholic steatohepatitis among a largely middle-aged population utilizing ultrasound and liver biopsy: a prospective study. Gastroenterology. 2011; 140:124-131. [PubMed: 20858492]

(69). Centers for Disease Control and Prevention (CDC). Hepatocellular carcinoma - United States, 2001-2006. MMWR Morb Mortal Wkly Rep. 2010; 59:517-520. [PubMed: 20448528] 

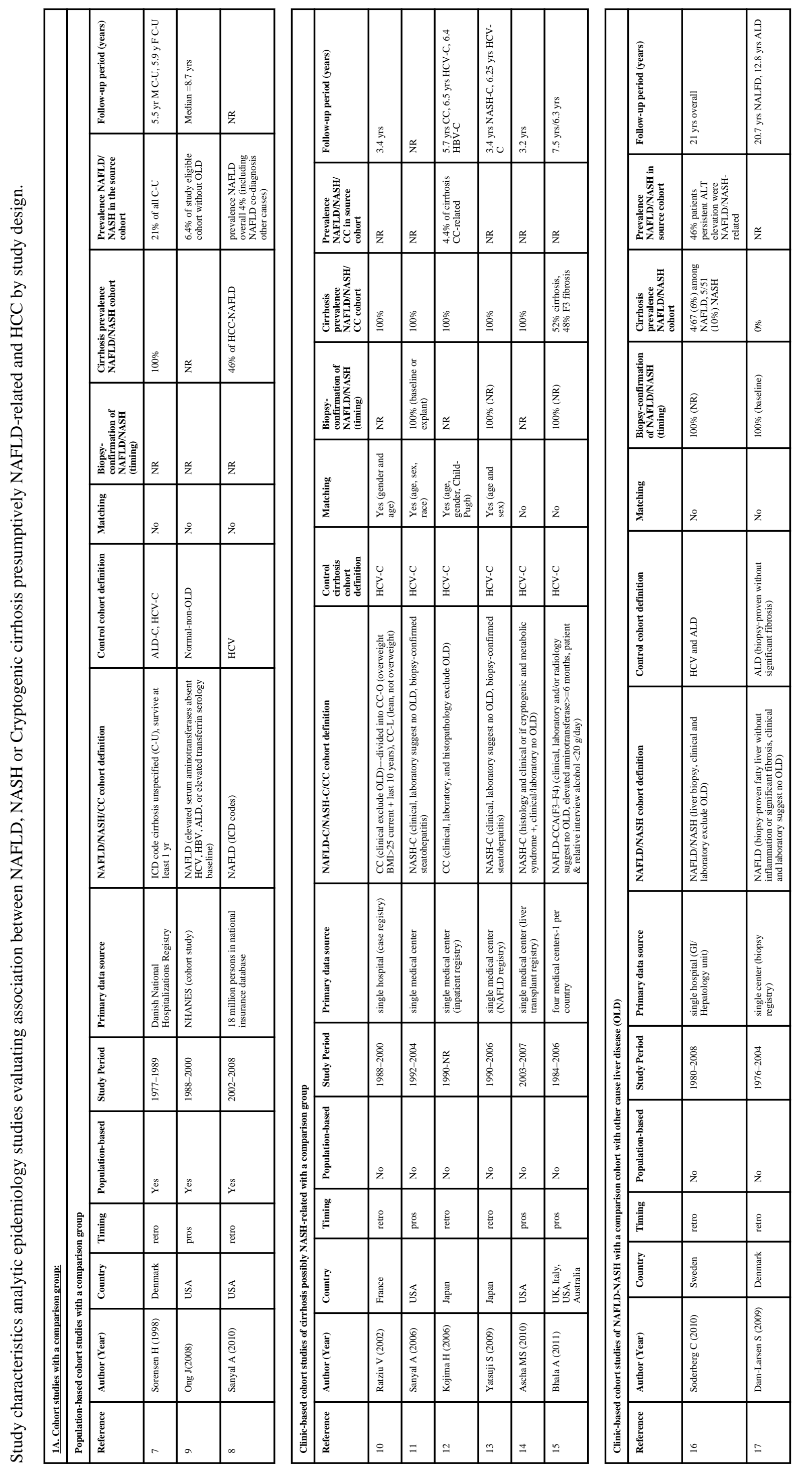

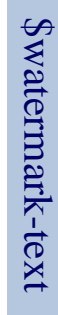
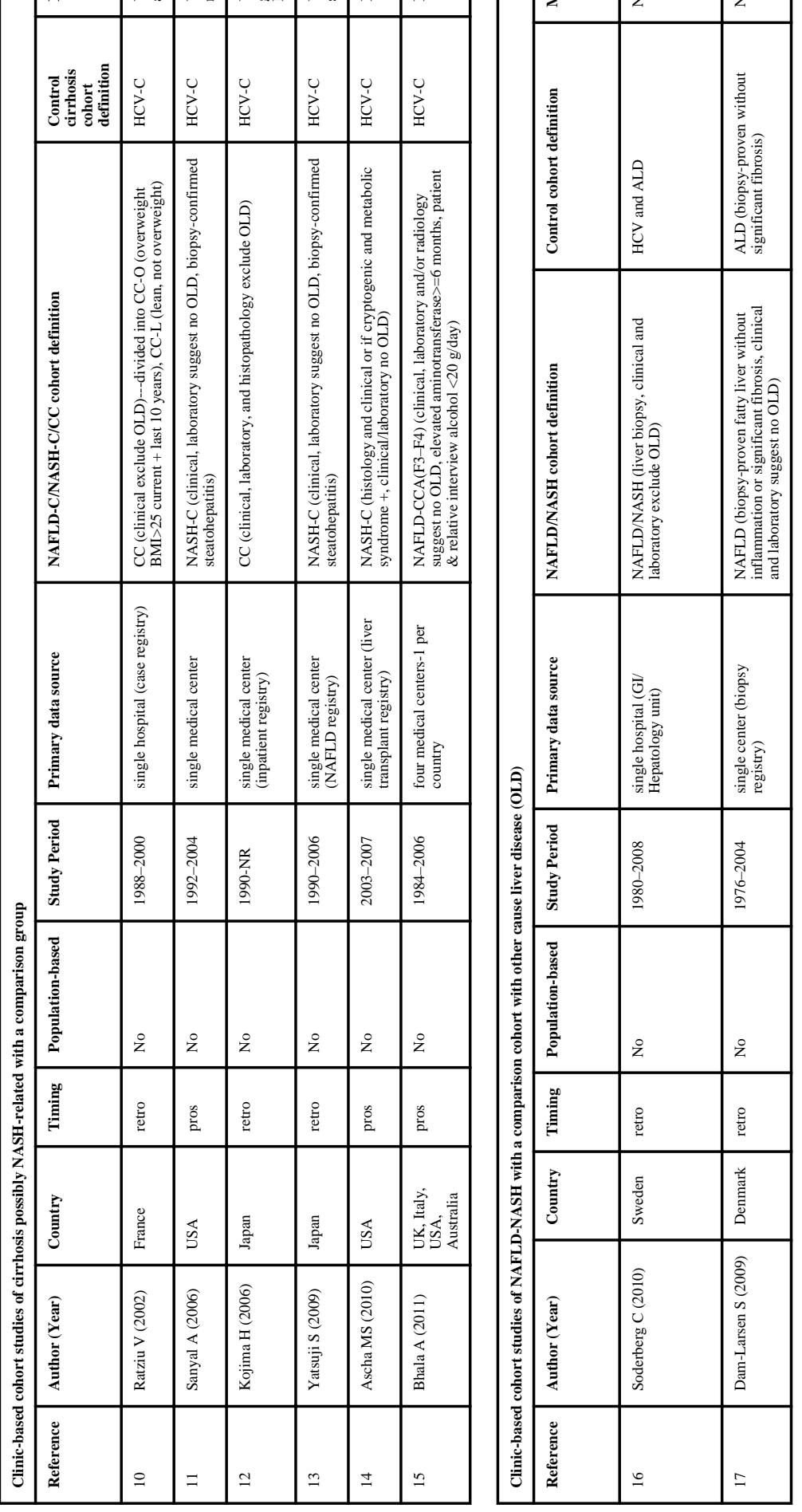

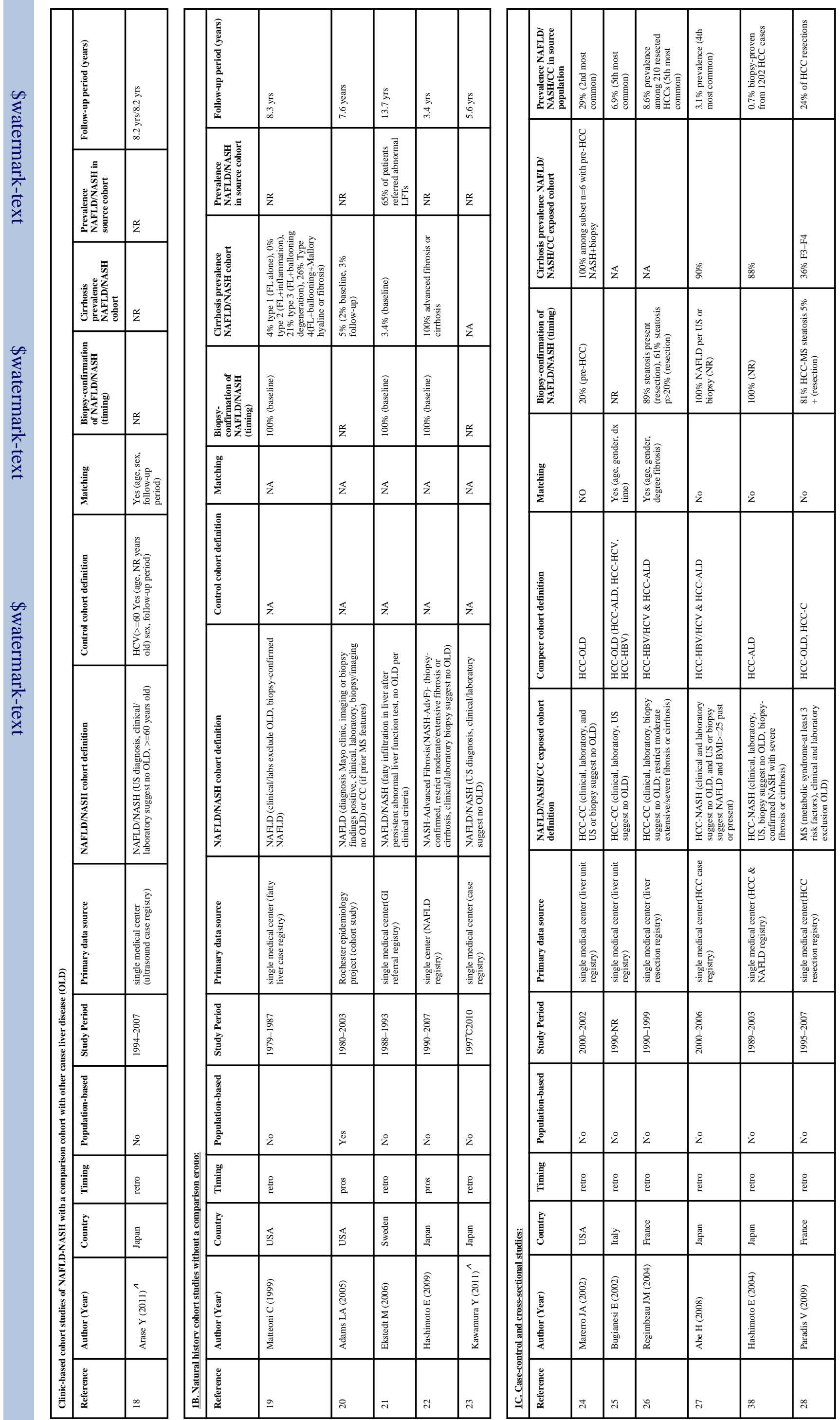


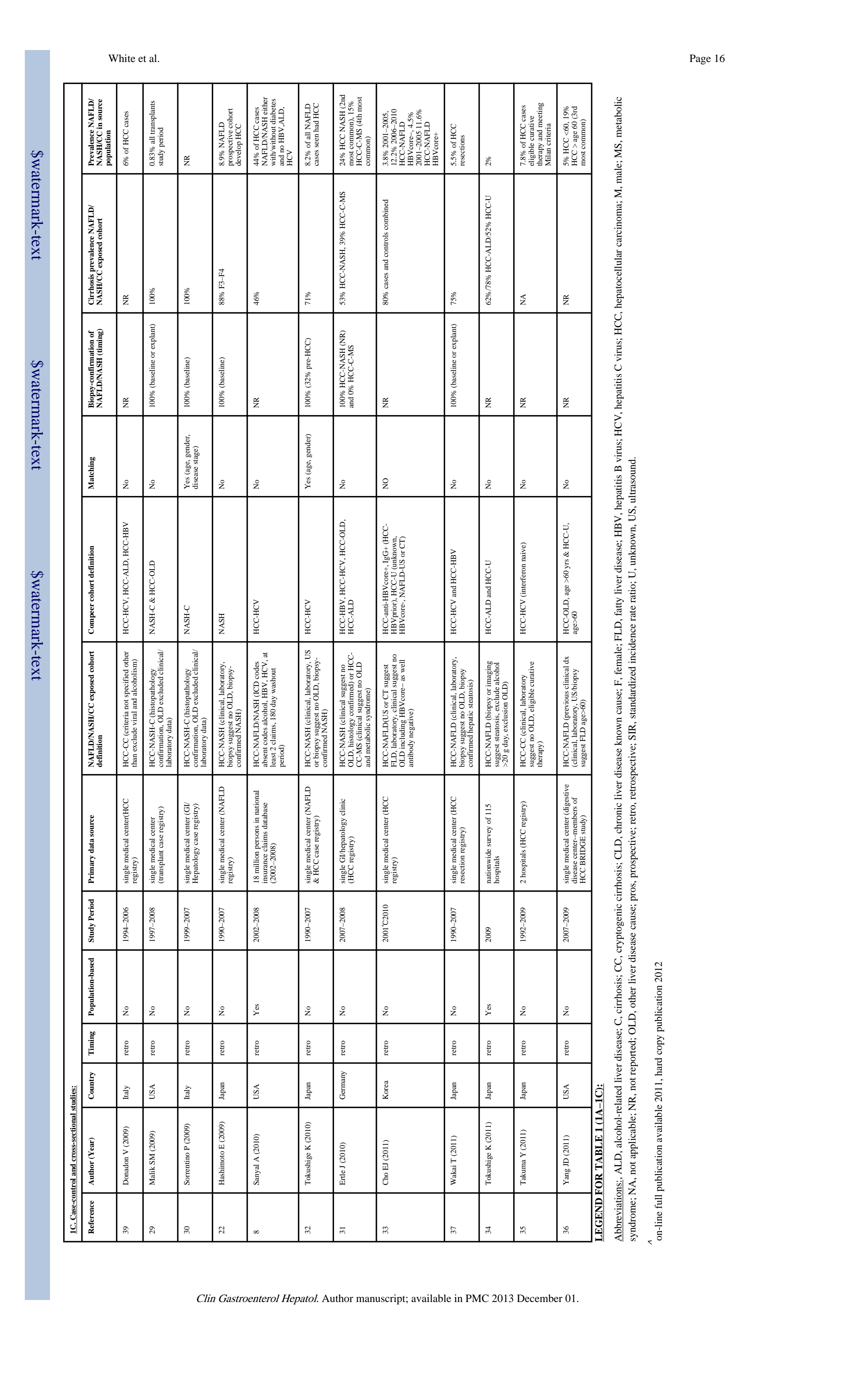




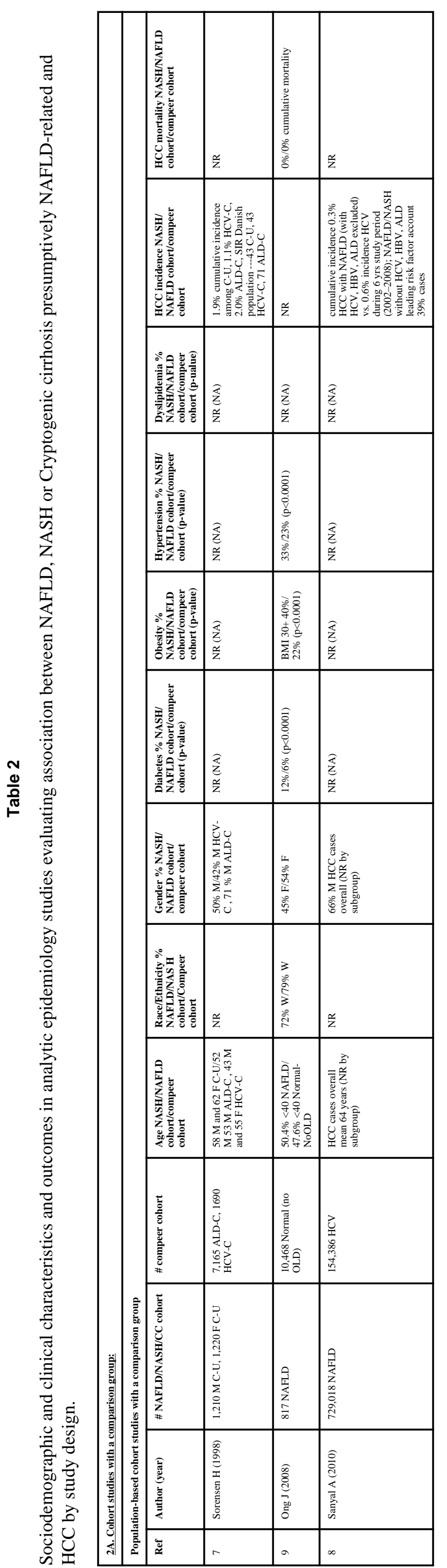

\begin{tabular}{|c|c|c|c|}
\hline 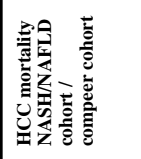 & $\tilde{\bar{z}}$ & 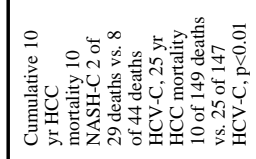 & 艺 \\
\hline 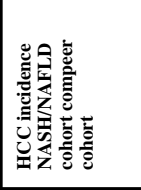 & 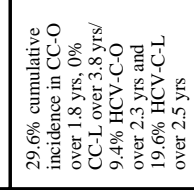 & 芒 & 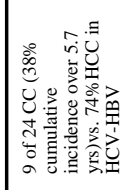 \\
\hline 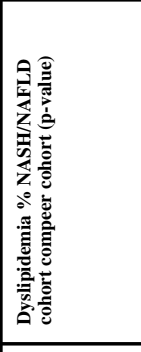 & 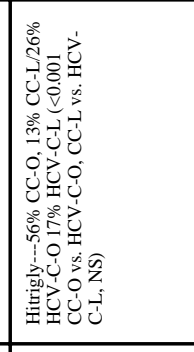 & 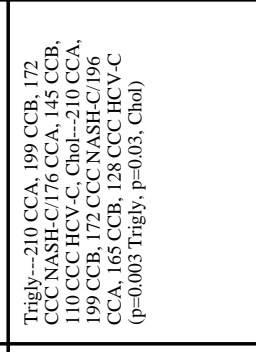 & 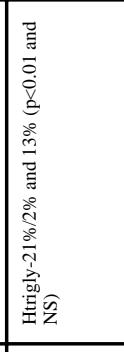 \\
\hline 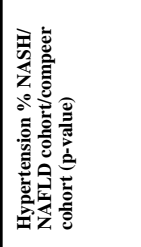 & $\mid \begin{array}{l}\frac{\pi}{3} \\
\frac{\tilde{z}}{\tilde{z}}\end{array}$ & 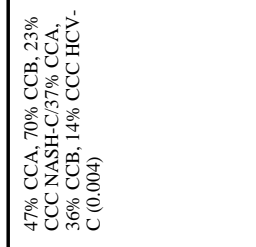 & 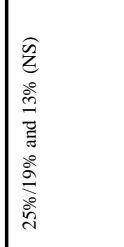 \\
\hline 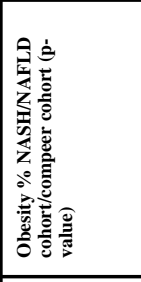 & 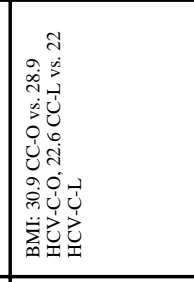 & 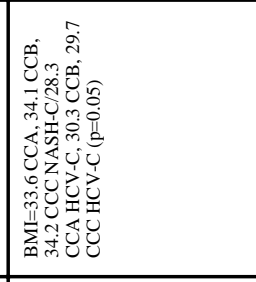 & 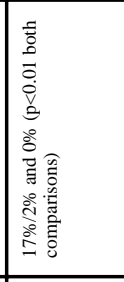 \\
\hline 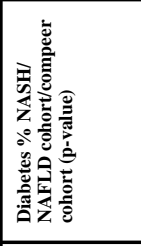 & 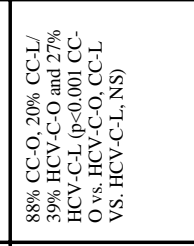 & 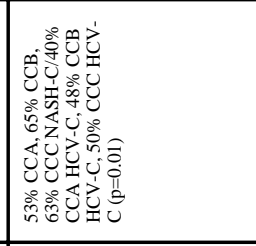 & 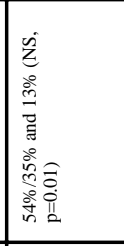 \\
\hline 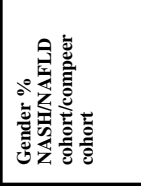 & 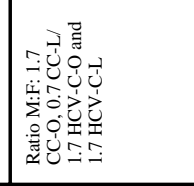 & 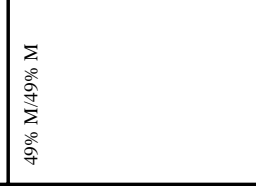 & 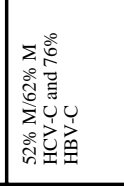 \\
\hline 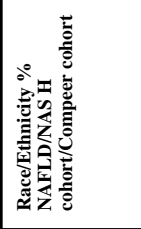 & 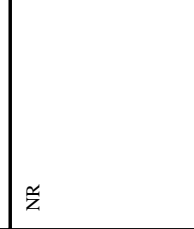 & 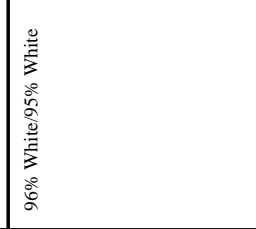 & 㕕 \\
\hline 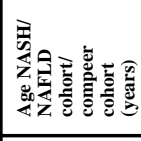 & 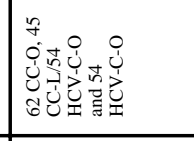 & 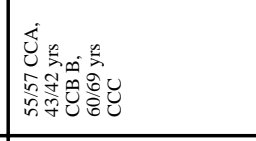 & 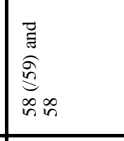 \\
\hline 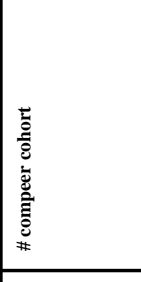 & 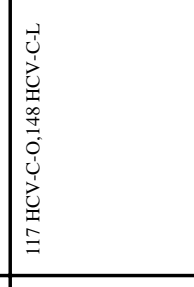 & 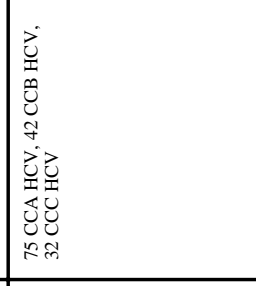 & 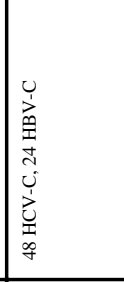 \\
\hline 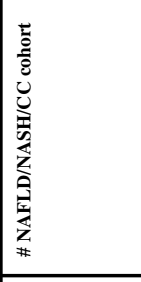 & 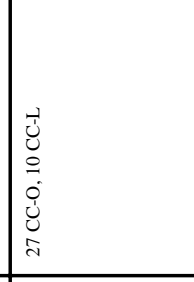 & 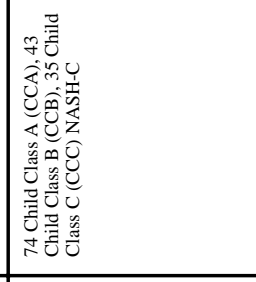 & 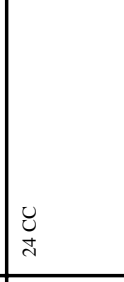 \\
\hline 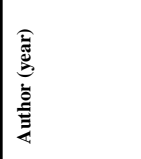 & 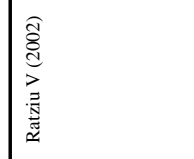 & 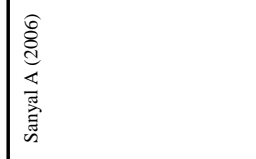 & 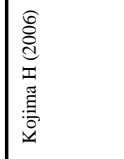 \\
\hline$\Xi$ & $\cong$ & $=$ & $\simeq$ \\
\hline
\end{tabular}





\begin{tabular}{|c|c|c|c|c|c|}
\hline 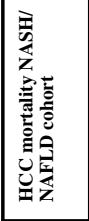 & 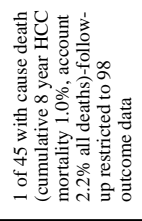 & 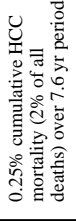 & 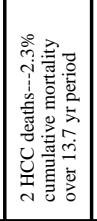 & 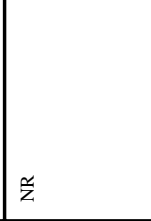 & $\tilde{\Sigma}$ \\
\hline 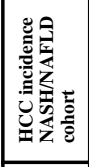 & 乏 & 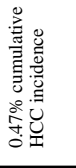 & $\frac{\pi}{z}$ & 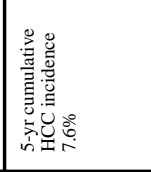 & 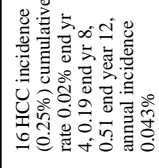 \\
\hline 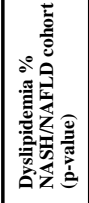 & 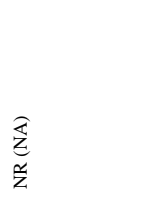 & 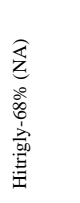 & 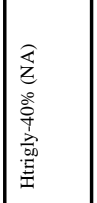 & 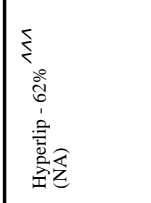 & 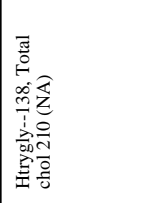 \\
\hline 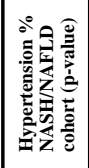 & 辰 & 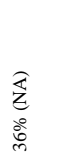 & 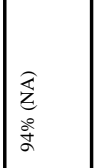 & 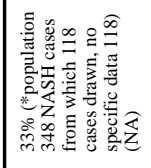 & 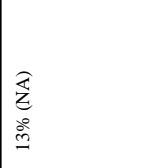 \\
\hline 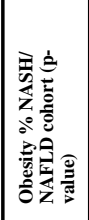 & 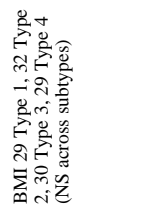 & 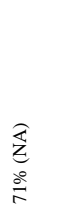 & 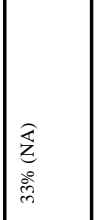 & 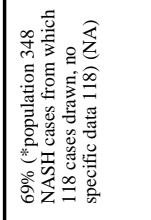 & 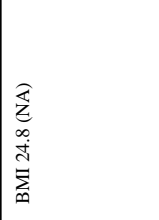 \\
\hline 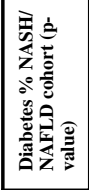 & 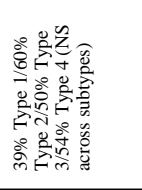 & 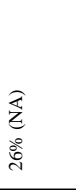 & 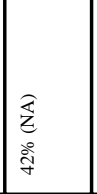 & 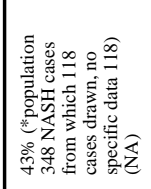 & 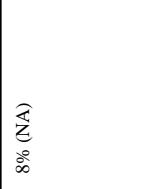 \\
\hline 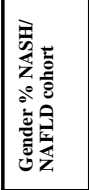 & 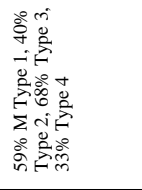 & 龒 & 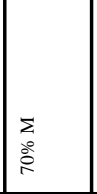 & 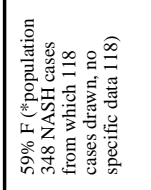 & 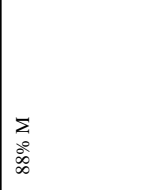 \\
\hline 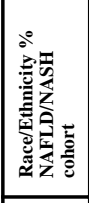 & 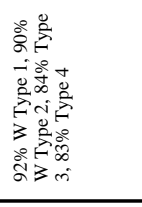 & s. & 芒 & 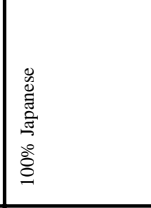 & $\frac{\pi}{2}$ \\
\hline 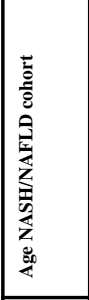 & 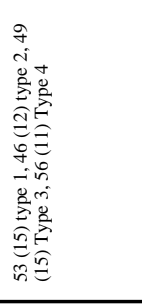 & 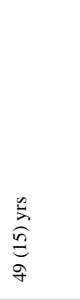 & 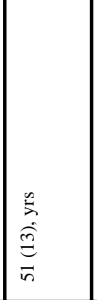 & 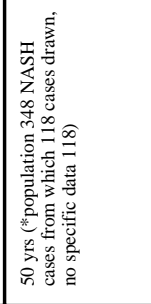 & $\begin{array}{l}\mathscr{\omega} \\
\text { d. } \\
\sigma \\
\sigma\end{array}$ \\
\hline 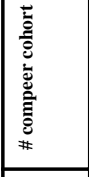 & $\frac{s}{z}$ & $\underline{z}$ & $\frac{\hat{z}}{\mathrm{z}}$ & $\frac{s}{z}$ & $\bar{z}$ \\
\hline 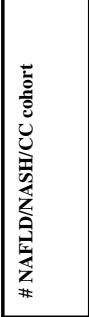 & 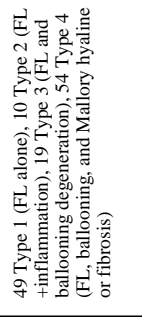 & 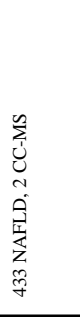 & 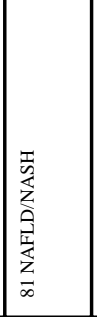 & 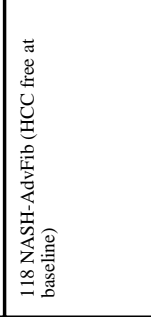 & 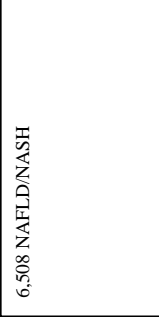 \\
\hline 焉 & 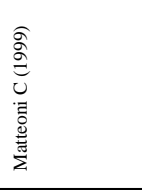 & 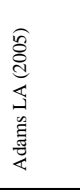 & $\mid$ & 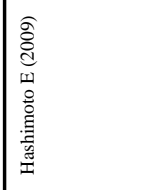 & 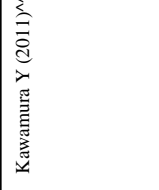 \\
\hline 㐫 & $\stackrel{9}{1}$ & $\therefore$ & $\overline{4}$ & z & $\approx$ \\
\hline
\end{tabular}

\begin{tabular}{|c|c|c|c|c|c|c|c|}
\hline 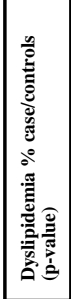 & 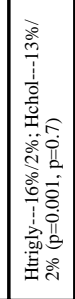 & 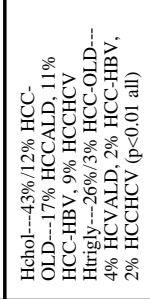 & 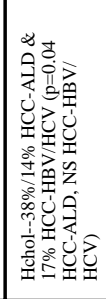 & 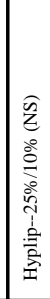 & 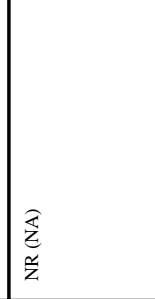 & $\mid \begin{array}{l}\hat{\bar{z}} \\
\frac{\tilde{z}}{\bar{z}}\end{array}$ & 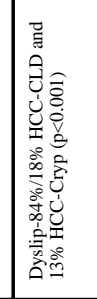 \\
\hline 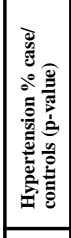 & 焉 & 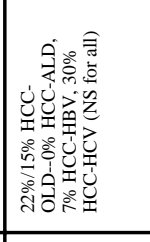 & 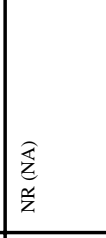 & 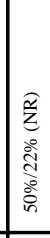 & 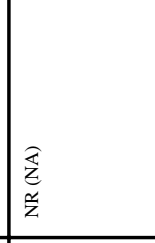 & \begin{tabular}{|l}
$\mid \frac{\pi}{\tilde{z}}$ \\
$\frac{\tilde{z}}{\tilde{z}}$
\end{tabular} & 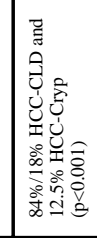 \\
\hline 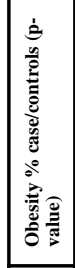 & 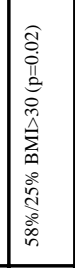 & 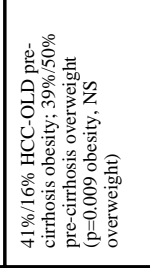 & 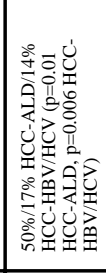 & 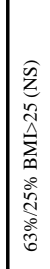 & 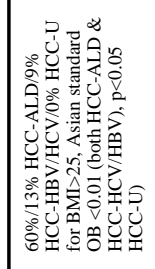 & 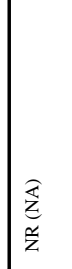 & 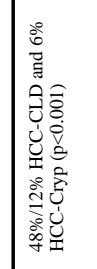 \\
\hline 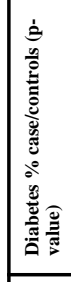 & 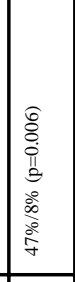 & 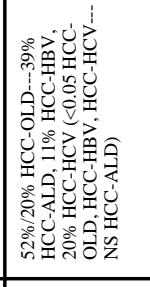 & 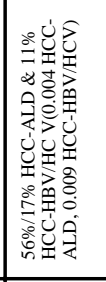 & 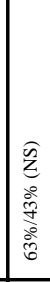 & 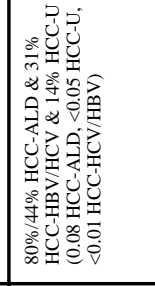 & 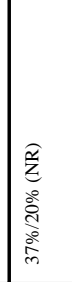 & 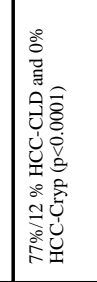 \\
\hline 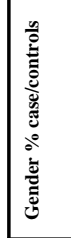 & 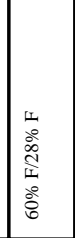 & 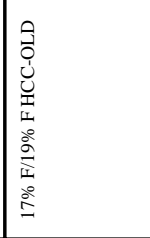 & 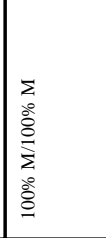 & 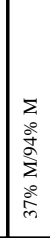 & 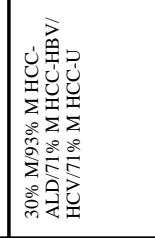 & 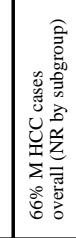 & 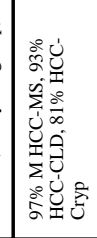 \\
\hline 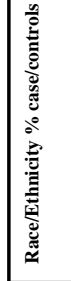 & 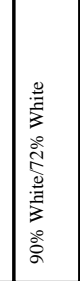 & 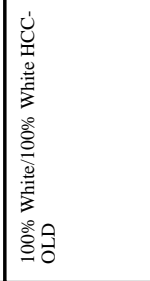 & 苂 & $\tilde{z}$ & 艺 & 芒 & 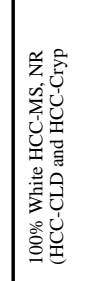 \\
\hline 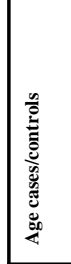 & 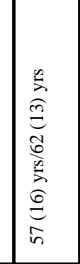 & 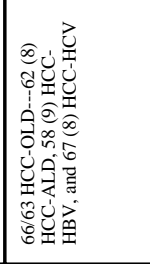 & 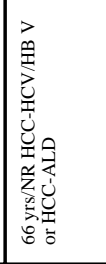 & 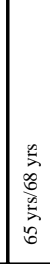 & 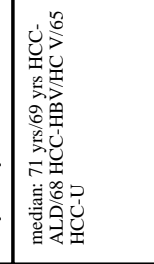 & 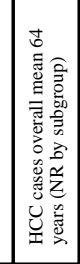 & 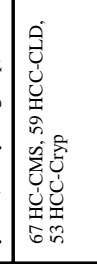 \\
\hline 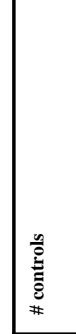 & 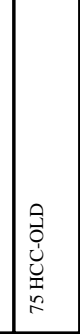 & 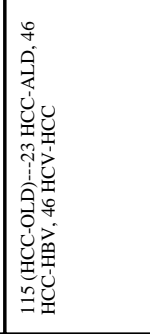 & 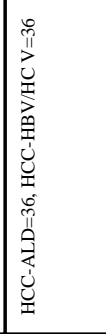 & 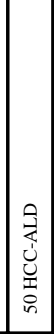 & 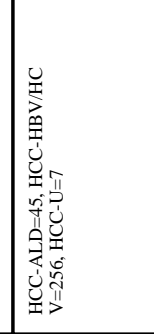 & 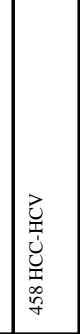 & 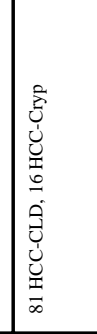 \\
\hline 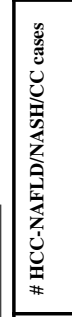 & 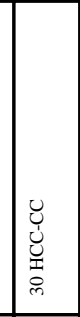 & 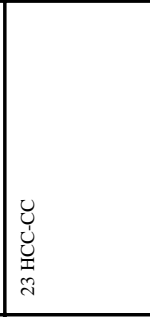 & 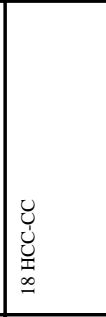 & 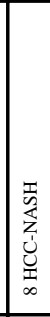 & 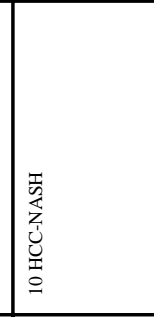 & 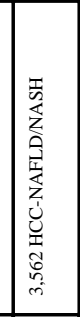 & 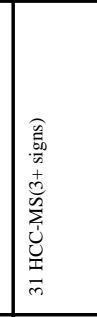 \\
\hline 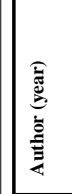 & 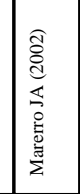 & 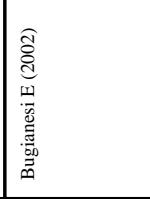 & 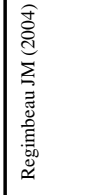 & 咅 & 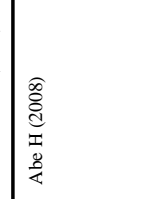 & 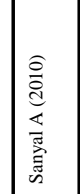 & 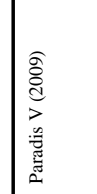 \\
\hline$\Xi$ & 吉 & 2 & $\therefore$ & $\therefore$ & $\infty$ & 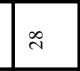 & $\%$ \\
\hline
\end{tabular}


